\title{
A Least-Squares FEM for the Direct and Inverse Rectangular Cavity Scattering Problem
}

\author{
Enxi Zheng, ${ }^{1}$ Fuming $\mathrm{Ma}^{2}$ and Yujie Wang ${ }^{2}$ \\ ${ }^{1}$ Department of Mathematics, Dalian Maritime University, Dalian 116026, China \\ ${ }^{2}$ School of Mathematics, Jilin University, Changchun 130012, China \\ Correspondence should be addressed to Enxi Zheng; enxizheng2003@163.com
}

Received 29 September 2014; Revised 14 February 2015; Accepted 14 February 2015

Academic Editor: Stefano Lenci

Copyright (c) 2015 Enxi Zheng et al. This is an open access article distributed under the Creative Commons Attribution License, which permits unrestricted use, distribution, and reproduction in any medium, provided the original work is properly cited.

\begin{abstract}
This paper is concerned with the scattering problem of a rectangular cavity. We solve this problem by a least-squares nonpolynomial finite element method. In the method, we use Fourier-Bessel functions to capture the behaviors of the total field around corners. And the scattered field towards infinity is represented by a combination of half-space Green functions. Then we analyze the convergence and give an error estimate of the method. By coupling the least-squares nonpolynomial finite element method and the Newton method, we proposed an algorithm for the inverse scattering problem. Numerical experiments are presented to show the effectiveness of our method.
\end{abstract}

\section{Introduction}

In recent years the scattering theory in electromagnetic cavity problem is very popular in mathematical physics and plays an important role in practical applications, for example, the radar detecting. Radar cross section (RCS) is a measure of the detectability of a target by a radar system and the RCS by a cavity is usually significant in overall RCS of an object. So the accurate prediction of the RCS of a cavity, that is, the electromagnetic field scattered by a cavity, attracts many scientists' interests. The cavity scattering theory can also be used to detect cracks or holes in metallic surfaces such as the aircraft wings. These cracks or holes may be invisible to a visual inspection but detectable by electromagnetic waves, so the understanding of scattering theory of cavity can help aerodynamicist to design and check the aircraft wings. In reality the engineers often use electromagnetic waves to check the material. Then the inverse scattering problem of a cavity can serve as a mathematical model.

For the scattering problem of an open cavity embedded in an infinite ground plane, Ammari et al. in $[1,2]$ reformulated the problem into a bounded domain via a variational approach and gave the existence and uniqueness results in two and three dimensions. In [3], Ammari et al. investigated the integral equations method to solve this problem and gave the existence and uniqueness results of the solution to the corresponding integral equations. Other numerical methods used to solve the scattering problem of open cavity are time-domain finite difference methods [4], finite element methods $[5,6]$, several hybrid methods [7-9], and so forth. Recently, in [10] Bao et al. analyzed the stability of the scattering from a large rectangular cavity and their stability estimates provided the explicit dependence on the high wave number and the depth of the cavity. Although the result in [10] is not optimal, it is essential for conducting convergence analysis of numerical methods. In [11] Li et al. also presented some stability estimates with the explicit dependency of wave number for the open cavity problem. And they proposed a Legendre spectral Galerkin method for the scattering problem of rectangular cavity. About the inverse scattering problem of cavity, we refer to $[12,13]$ and the references therein. In [13] the author recovered the shape of the cavity from the scattered field given in the aperture and gave the conditions needed for proving the uniqueness of this inverse problem. In [12], Feng and Ma investigated the inverse problem of determining the shape of the open cavity from the information of the far field patterns of scattered field. The results on the uniqueness and the local stability 
of the inverse problem in the 2-dimensional TM (transverse magnetic) polarization were also proved in the paper. Lately, Bao et al. studied the direct and inverse problem of open cavity in TM and TE (transverse electric) polarization. In [14] they proposed a method of symmetric coupling of finite element and boundary integral equations to solve the direct problem and proved the existence and uniqueness of weak solutions. As for inverse problem, the domain derivatives of the field with respect to the shape of the cavity were derived and the uniqueness and local stability results were established.

In [15], the authors proposed a nonpolynomial finite element method for the scattering problem of a polygonal obstacle. This scattering problem is defined in the whole $\mathbb{R}^{2}$ except the obstacle domain. In this paper, we consider the scattering problem of a rectangular open cavity. This scattering problem is defined in the whole upper half-plane and cavity domain which embedded in the lower halfplane. Inspired by [15], we solve this scattering problem of a rectangular cavity by the least-squares nonpolynomial finite element method. In case of the obstacle scattering problem, the scattering field towards infinity is represented by a combination of free-space Green functions, but in case of our open cavity scattering problem, the scattering field towards infinity is represented by a combination of half-space Green functions. Furthermore, in [15] the authors only gave the approximation properties of finite element space. In our paper we give the $L^{2}$ error estimate using the approximation properties in [15] and dual technique in [16]. The difference between [16] and our paper is that we solve the Helmholtz equation in semi-infinite domain and the authors in [16] were concerned with the Helmholtz problem in bounded domain.

In $[12,14]$ the existence, uniqueness, and local stability of inverse scattering problem for open cavity are proved. Here, the local stability means when the Hausdorff distance between two cavities is not too big, this distance can be controlled by the difference of the scattered fields for these two cavities. But in the above two papers, the authors did not present a numerical method for inverse cavity problem. In our paper we propose a Newton method coupling with the least-squares finite element method to solve the inverse scattering problem of open cavity. In order to implement the Newton method we should get the domain derivative, that is, the derivative of scattered field about the domain parameters. Paper [17] gave the general theory of domain derivative for boundary value problem. References $[18,19]$ presented the differential equation which the domain derivative satisfies in inverse obstacle problem. With the help of these results we give the domain derivative of the inverse scattering problem for rectangular cavity and use it in the numerical algorithm.

In this paper, we consider the scattering problem of an open cavity with a rectangular cross section and assume that the cross section of the rectangular cavity is under $y=0$. Let $D$ be a rectangular domain given by $D:=(a, a+l) \times(-h, 0)$, where $a<0, l>|a|$, and $h>0$. Denote the upper halfplane by $\mathbb{R}_{+}^{2}:=\left\{\mathbf{x}=(x, y) \in \mathbb{R}^{2}, y>0\right\}$, and define $\Sigma:=$ $(\partial D \backslash[a, a+l] \times\{0\}) \cup(-\infty, a] \times\{0\} \cup[a+l,+\infty) \times\{0\} ;$ see Figure 1 . By this definition, a rectangular cavity $D$ can be determined by three parameters $a, l$, and $h$, where $(a, 0)$ is

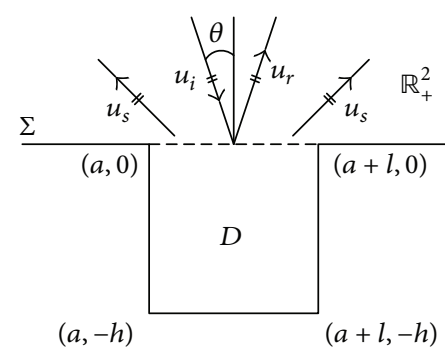

FIGURE 1: Cavity domain.

the left tip of the opening aperture, $l$ is the width of the cavity, and $h$ is the depth of the cavity.

It is assumed that the medium for $\mathbb{R}_{+}^{2} \cup D$ is homogeneous, and the cavity is on a perfect conducting ground. In the TM polarization, we assume that $u_{i}=e^{i \alpha x-i \beta y}$ is the incident field, where $\alpha=k \sin \theta, \beta=k \cos \theta, \theta \in(-\pi / 2, \pi / 2)$ is the angle of incidence with respect to $y$-axis, and $k$ is the wave number. The total field $u$ consists of three parts:

$$
u=u_{i}+u_{r}+u_{s}
$$

where $u_{r}=-e^{i \alpha x+i \beta y}$ and $u_{s}$ is the scattering field. Then the scattering problem reads as follows: given incident plane wave $u_{i}$, seek a solution $u$ such that

$$
\begin{gathered}
\Delta u+k^{2} u=0 \quad \text { in } D \cup \mathbb{R}_{+}^{2}, \\
u=0, \quad \text { on } \Sigma, \\
\lim _{r \rightarrow \infty} \sqrt{r}\left(\partial_{r} u_{s}-i k u_{s}\right)=0 .
\end{gathered}
$$

The third equation in (2) is the Sommerfeld radiation condition which guarantees that the scattering wave is outgoing, where $r=|\mathbf{x}|$. The analysis of this problem can be found in [1], where the existence and the uniqueness of the solution were proved.

In this paper, we solve the above problem by a leastsquares nonpolynomial finite element method. We use Fourier-Bessel functions and half-space Green functions to construct the bases of our method. These bases capture the behaviors of the total field around the corner and the behaviors of the scattering field towards infinity very well. So our numerical method is very efficient and highly accurate.

The outline of the paper is as follows. In Section 2, the least-squares nonpolynomial finite element method for the direct scattering problem of rectangular open cavity is stated. In Section 3, we show the convergence and $L^{2}$ error estimate for the method. In Section 4, we propose an algorithm for the inverse scattering problem of rectangular open cavity by coupling the least-squares nonpolynomial finite element method and Newton method. Finally, several numerical examples are included to show the effectiveness of our method.

\section{Formulation of the Method}

Let $R>\max \{|a|,|a+l|\}, \mathbf{x}=(x, y) \in \mathbb{R}^{2}, \Gamma_{R}=\{|\mathbf{x}|=R, y \geq$ $0\}, \Gamma=\{\mathbf{x} \in \Sigma,|x|<R\}$, and $E=\{|\mathbf{x}|<R, y>0\} \cup D$. 


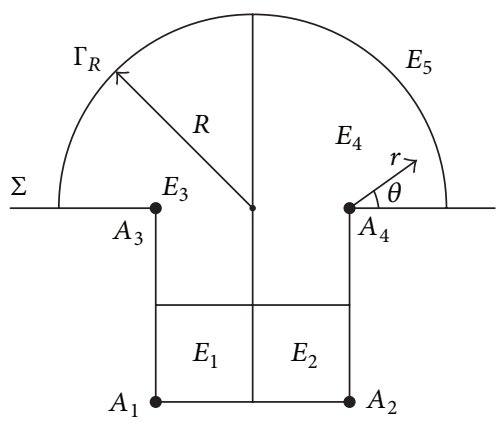

Figure 2: Cavity domain and decomposition.

$\left\{E_{1}, E_{2}, E_{3}, E_{4}\right\}$ is a decomposition of domain $E$ such that each $E_{j}, j=1, \ldots, 4$, contains a corner of $\Sigma$. Denote $E_{5}=\left(\mathbb{R}_{+}^{2} \cup\right.$ D) $\backslash \bar{E}$ and then the whole semi-infinite domain $\mathbb{R}_{+}^{2} \cup D$ is divided into five subdomains: $E_{1}, E_{2}, E_{3}, E_{4}, E_{5}$; see Figure 2 .

As is done in [15], in each $E_{j}, j=1, \ldots, 4$, the total field $u$ can be approximated by

$$
u(r, \theta) \approx \sum_{n=1}^{N_{j}} c_{n}^{(j)} J_{\gamma_{j} n}(k r) \sin \left(\gamma_{j} n \theta\right),
$$

where $N_{j} \in \mathbb{N}, \gamma_{1}=\gamma_{2}=2, \gamma_{3}=\gamma_{4}=2 / 3,(r, \theta)$ is the local polar coordinate (see Figure 2), and $J_{\gamma_{j} n}$ is the Bessel function of order $\gamma_{j} n$.

Denote the half-space Green function of Helmholtz equation by $G(\mathbf{x}, \mathbf{y})$; that is,

$$
G(\mathbf{x}, \mathbf{y})=\frac{i}{4}\left(H_{0}^{(1)}(k|\mathbf{x}-\mathbf{y}|)-H_{0}^{(1)}\left(k\left|\mathbf{x}-\mathbf{y}^{\prime}\right|\right)\right),
$$

where $\mathbf{x}, \mathbf{y} \in \mathbb{R}_{+}^{2}$, and $\mathbf{y}^{\prime}$ is the reflection of $\mathbf{y}$ in $x$-axis (i.e., if $\mathbf{y}=\left(y_{1}, y_{2}\right)$, and then $\left.\mathbf{y}^{\prime}=\left(y_{1},-y_{2}\right)\right)$. Let $0<R_{0}<R$, $\Gamma_{R_{0}}=\left\{|\mathbf{x}|=R_{0}, x_{2} \geq 0\right\}$. Choose $\mathbf{y}_{j}=R_{0} e^{i \phi_{j}} \in \Gamma_{R_{0}}$, and $\phi_{j}=j \pi / N_{5}, j=1,2, \ldots, N_{5}-1, N_{5} \in \mathbb{N}$. In $E_{5}$, the scattering field $u_{s}$ can be approximated by a linear combination of the half-space Green functions, so the total field $u$ can be approximated by

$$
\begin{array}{r}
u \approx u_{i}+u_{r}+\sum_{j=1}^{N_{5}-1} c_{j}^{(5)}\left(\frac{\partial}{\partial n\left(\mathbf{y}_{j}\right)} G\left(\mathbf{x}, \mathbf{y}_{j}\right)+i k G\left(\mathbf{x}, \mathbf{y}_{j}\right)\right), \\
\mathbf{x} \in E_{5} .
\end{array}
$$

Denote

$$
\begin{aligned}
& V_{j}=\left\{v \in L^{2}\left(E_{j}\right), v=\sum_{n=1}^{N_{j}} c_{n}^{(j)} J_{\gamma_{j} n}(k r) \sin \left(\gamma_{j} n \theta\right)\right\}, \\
& j=1,2,3,4 \text {; } \\
& V_{5}=\left\{v \in L_{\text {loc }}^{2}\left(E_{5}\right), v=u_{i}+u_{r}\right. \\
& \left.+\sum_{j=1}^{N_{5}-1} c_{j}^{(5)}\left(\frac{\partial}{\partial n\left(\mathbf{y}_{j}\right)} G\left(\mathbf{x}, \mathbf{y}_{j}\right)+i k G\left(\mathbf{x}, \mathbf{y}_{j}\right)\right)\right\},
\end{aligned}
$$

then our trial space $V$ can be defined as follows:

$$
V=\prod_{j=1}^{5} V_{j}
$$

We denote the boundary of $E_{j}$ by $\Gamma_{j}, j=1,2,3,4$, and $\Gamma_{5}=\Gamma_{R}$. Let $\Gamma_{i, j}=\Gamma_{i} \cap \Gamma_{j}, i, j=1,2, \ldots, 5$, and $n(\mathbf{x}), \mathbf{x} \in \Gamma_{i, j}$, be a unit normal of $\Gamma_{i, j}$. If $i<j$, then $n(x)$ points from $E_{i}$ to $E_{j}$; if $i>j$, then $n(x)$ points from $E_{j}$ to $E_{i}$. Before we explain the least-squares finite element formulation as proposed by Stojek in [20], Monk and Wang in [16], and Barnett and Betcke in [15], we need to define the jump of $v \in V$ on $\Gamma_{i, j}$. For a function $v \in V$, the jump of $v$ on $\Gamma_{i, j}$ is defined as follows:

$$
\begin{array}{r}
{[v](x):=\lim _{\varepsilon \rightarrow 0} v(\mathbf{x}+\varepsilon n(\mathbf{x}))-v(\mathbf{x}-\varepsilon n(\mathbf{x})),} \\
\mathbf{x} \in \Gamma_{i, j} .
\end{array}
$$

The jump of normal derivative is defined in the same way and denoted by $\left[\partial_{n} v\right]$. In order to enforce the continuity on all interfaces $\Gamma_{i, j}(i<j)$, we define the following functional:

$$
J(v):=\sum_{i<j} \int_{\Gamma_{i, j}}\left|\left[\partial_{n} v\right]\right|^{2}+k^{2}|[v]|^{2} \mathrm{~d} s .
$$

The least-squares finite element approximation $u_{N}$ is now defined as the solution of the following least-squares problem:

$$
u_{N}:=\arg \min _{v \in V} J(v) .
$$

\section{Error Estimate}

In this section we give an $L^{2}$ error estimate for our leastsquares finite element method. First, we restrict problem (2) from infinite domain to a bounded domain. Next, using the technique presented in [16], we provide a basic error estimate for the method. Then, we apply the basic error estimate together with approximation properties of the basis functions to prove the desired error estimate. In this section and the following sections, $C$ denotes a generic constant, which may have different values at different places.

Let $R_{2}>R$ and $\widehat{E}_{5}=\left\{\mathbf{x} \in \mathbb{R}_{+}^{2}, R<|\mathbf{x}|<R_{2}\right\}$. We start by introducing an equivalent formulation of (2) in the bounded domain $E_{R_{2}}=E \cup \widehat{E}_{5}$. In domain $\mathbb{R}_{+}^{2} \backslash E_{R_{2}}$, the scattering field $u_{s}$ can be written under the polar coordinates as follows:

$$
\begin{array}{r}
u_{s}(r, \theta)=\sum_{n=1}^{\infty} u_{s}^{(n)} \frac{H_{n}^{(1)}(k r)}{H_{n}^{(1)}\left(k R_{2}\right)} \sin (n \theta), \\
r \geq R_{2}, \quad \theta \in[0, \pi],
\end{array}
$$

where $H_{n}^{(1)}$ is the Hankel function of the first kind of order $n$, and

$$
u_{s}^{(n)}=\frac{2}{\pi} \int_{0}^{\pi} u^{s}\left(R_{2}, \theta^{\prime}\right) \sin \left(n \theta^{\prime}\right) \mathrm{d} \theta^{\prime} .
$$

Since $u^{s}$ is analytic in $E_{5}$, from the decay of Fourier coefficient for analytic function, it holds that there exists a $\rho>0$ such that

$$
\left|u_{s}^{(n)}\right|=O\left(\rho^{-n}\right)
$$



$u_{s}:$

By simple calculation, we can get the following relation of

$$
\frac{\partial u_{s}}{\partial n}=T u_{s}, \quad \text { on } \Gamma_{R_{2}},
$$

where $T$ is the Dirichlet to Neumann operator defined as follows: for any function $f \in H^{1 / 2}\left(\Gamma_{R}\right)$ which has the expansion $f=\sum_{n=1}^{\infty} f^{(n)} \sin (n \theta)$,

$$
\begin{gathered}
T f=\sum_{n \in \mathbb{Z}} k \frac{H_{n}^{(1) \prime}\left(k R_{2}\right)}{H_{n}^{(1)}\left(k R_{2}\right)} f^{(n)} \sin (n \theta), \\
f^{(n)}=\frac{2}{\pi} \int_{0}^{\pi} f \sin (n \theta) \mathrm{d} \theta .
\end{gathered}
$$

Denote $\Sigma_{R_{2}}=\left\{\mathbf{x}=\left(x_{1}, x_{2}\right) \in \Sigma, x_{1} \leq R_{2}\right\}$ and $\Gamma_{R_{2}}=\{\mathbf{x} \in$ $\left.\mathbb{R}_{+}^{2},|\mathbf{x}|=R_{2}\right\}$. Using the Dirichlet to Neumann operator $T$, problem (2) can be restricted to domain $E_{R_{2}}$; that is, given incident plane wave $u_{i}$, find a solution $u$ such that

$$
\begin{gathered}
\Delta u+k^{2} u=0, \quad \text { in } E_{R_{2}}, \\
u=0, \quad \text { on } \Sigma_{R_{2}}, \\
\frac{\partial u_{s}}{\partial n}=T u_{s}, \quad \text { on } \Gamma_{R_{2}} .
\end{gathered}
$$

Theorem 1. Assuming that $u_{N}$ is the solution of the leastsquares problem (10), then there exists a constant $C$ such that

$$
\left\|u-u_{N}\right\|_{0, E_{R_{2}}} \leq C J\left(u_{N}\right)^{1 / 2} .
$$

Proof. Let $v$ satisfy the dual problem

$$
\begin{gathered}
\Delta v+k^{2} v=\phi \quad \text { in } E_{R_{2}}, \\
v=0, \quad \text { on } \Sigma_{R_{2}}, \\
\frac{\partial v}{\partial n}=T^{*} v, \quad \text { on } \Gamma_{R_{2}},
\end{gathered}
$$

where $\phi$ is an arbitrary function in $L^{2}\left(E_{R_{2}}\right)$ and $T^{*}$ is the adjoint operator of $T$. Using Green's formula and the fact that in each $E_{j}(j=1, \ldots, 4)$ or $\widehat{E}_{5}, u_{N}$ is a solution of the Helmholtz equation, we can write

$$
\begin{aligned}
\left(u-u_{N}, \phi\right)_{E_{R_{2}}} & \left(u-u_{N}, \Delta v+k^{2} v\right)_{E_{R_{2}}} \\
= & \\
= & \sum_{j=1}^{4}\left(u-u_{N}, \Delta v+k^{2} v\right)_{E_{j}}+\left(u-u_{N}, \Delta v+k^{2} v\right)_{\widehat{E}_{5}} \\
= & \sum_{j=1}^{4}\left(\left(\Delta\left(u-u_{N}\right)+k^{2}\left(u-u_{N}\right), v\right)_{E_{j}}\right. \\
& \left.\quad+\left\langle u-u_{N}, \frac{\partial v}{\partial v_{j}}\right\rangle_{\partial E_{j}}-\left\langle\frac{\partial\left(u-u_{N}\right)}{\partial v_{j}}, v\right\rangle_{\partial E_{j}}\right) \\
& +\left(\Delta\left(u-u_{N}\right)+k^{2}\left(u-u_{N}\right), v\right)_{\widehat{E}_{5}}
\end{aligned}
$$

$$
\begin{gathered}
+\left\langle u-u_{N}, \frac{\partial v}{\partial \nu_{5}}\right\rangle_{\partial \widehat{E}_{5}}-\left\langle\frac{\partial\left(u-u_{N}\right)}{\partial v_{5}}, v\right\rangle_{\partial \widehat{E}_{5}} \\
=\sum_{j=1}^{4}\left(\left\langle u-u_{N}, \frac{\partial v}{\partial \nu_{j}}\right\rangle_{\partial E_{j}}-\left\langle\frac{\partial\left(u-u_{N}\right)}{\partial v_{j}}, v\right\rangle_{\partial E_{j}}\right) \\
+\left\langle u-u_{N}, \frac{\partial v}{\partial v_{5}}\right\rangle_{\partial \widehat{E}_{5}}-\left\langle\frac{\partial\left(u-u_{N}\right)}{\partial v_{5}}, v\right\rangle_{\partial \widehat{E}_{5}} .
\end{gathered}
$$

Here $\langle\cdot, \cdot\rangle_{S}$ is the $L^{2}(S)$ inner product (or duality pairing as appropriate) and $v_{j}(j=1, \ldots, 4)$ or $v_{5}$ is the unit outward normal to $E_{j}, \quad(j=1, \ldots, 4)$ or $\widehat{E}_{5}$.

Since

$$
\begin{gathered}
\left\langle u-u_{N}, T^{*} v\right\rangle_{\Gamma_{R_{2}}}=\left\langle T\left(u-u_{N}\right), v\right\rangle_{\Gamma_{R_{2}}}, \\
\left\langle\frac{\partial\left(u-u_{N}\right)}{\partial n}, v\right\rangle_{\Gamma_{R_{2}}}=\left\langle T\left(u-u_{N}\right), v\right\rangle_{\Gamma_{R_{2}}},
\end{gathered}
$$

using the boundary conditions for $u$ and $v$, substituting the above two identities in (19), and rewriting these sums as sums over edges we obtain

$$
\begin{aligned}
& \left(u-u_{N}, \phi\right) \\
& \quad=\sum_{i<j}\left(\left\langle\left[u-u_{N}\right], \frac{\partial v}{\partial n}\right\rangle_{\Gamma_{i, j}}-\left\langle\left[\frac{\partial\left(u-u_{N}\right)}{\partial n}\right], v\right\rangle_{\Gamma_{i, j}}\right) .
\end{aligned}
$$

Since the exact solution and its normal derivatives are continuous across $\Gamma_{i, j}$, we have

$$
\left(u-u_{N}, \phi\right)=\sum_{i<j}\left(\left\langle\left[u_{N}\right], \frac{\partial v}{\partial n}\right\rangle_{\Gamma_{i, j}}-\left\langle\left[\frac{\partial u_{N}}{\partial n}\right], v\right\rangle_{\Gamma_{i, j}}\right) .
$$

Hence, using the Cauchy-Schwarz inequality we obtain

$$
\begin{gathered}
\left|\left(u-u_{N}, \phi\right)\right| \leq \sum_{i<j}\left(\left\|\left[u_{N}\right]\right\|_{0, \Gamma_{i, j}}\left\|\frac{\partial v}{\partial n}\right\|_{0, \Gamma_{i, j}}\right. \\
\left.+\left\|\left[\frac{\partial u_{N}}{\partial n}\right]\right\|_{0, \Gamma_{i, j}}\|v\|_{0, \Gamma_{i, j}}\right) \\
\leq\left\{\sum_{i<j}\left(k^{2}\left\|\left[u_{N}\right]\right\|_{0, \Gamma_{i, j}}^{2}+\left\|\left[\frac{\partial u_{N}}{\partial n}\right]\right\|_{0, \Gamma_{i, j}}^{2}\right)\right\}^{1 / 2} \\
\cdot\left\{\sum_{i<j}\left(\frac{1}{k^{2}}\left\|\frac{\partial v}{\partial n}\right\|_{0, \Gamma_{i, j}}^{2}+\|v\|_{0, \Gamma_{i, j}}^{2}\right)\right\}^{1 / 2}
\end{gathered}
$$

Hence, we obtain the bound

$$
\left|\left(u-u_{N}, \phi\right)\right| \leq J\left(u_{N}\right)^{1 / 2}|\|v \mid\|,
$$


where

$$
\|v\| \mid=\left\{\sum_{i<j}\left(\frac{1}{k^{2}}\left\|\frac{\partial v}{\partial n}\right\|_{0, \Gamma_{i, j}}^{2}+\|v\|_{0, \Gamma_{i, j}}^{2}\right)\right\}^{1 / 2} .
$$

Using the trace theorem and the regularity result of $v$, we have

$$
\|v\|\|\leq C\| v\left\|_{3 / 2+\varepsilon, \Omega} \leq C\right\| \phi \|_{0, \Omega},
$$

where $\varepsilon$ is small enough. Using this estimate in (24), we obtain the desired estimate.

Remark 2. Theorem 1 shows that the functional $J\left(u_{N}\right)$ controls the interior error. Next, we give an estimate of $J\left(u_{N}\right)$ to show the convergence of our method.

The following lemma is from [15]. A similar lemma can also be found in [21]. We refer to [21] (Section 5) for the proof.

Lemma 3. In each $E_{j}, j=1, \ldots, 4$, there exists $\rho_{j}>1$ such that for every $1<\tau<\rho_{j}$ it holds that

$$
\min _{v \in V_{j}}\|u-v\|_{L^{\infty}\left(E_{j}\right)}=O\left(\tau^{-N_{j}}\right)
$$

as $N_{j} \rightarrow \infty$. Furthermore, there exist functions $\widetilde{v}_{j} \in V_{j}$ such that $\left\|u-\widetilde{v}_{j}\right\|_{L^{\infty}\left(E_{j}\right)}=O\left(\tau^{-N_{j}}\right)$ and

$$
\left\|\nabla u-\nabla \widetilde{v}_{j}\right\|_{L^{\infty}\left(\Gamma_{j}\right)}=O\left(\tau^{-N_{j}}\right),
$$

for every $1<\tau<\rho_{j}$, as $N_{j} \rightarrow \infty$.

Using the above lemma, we have the following proposition immediately.

Proposition 4. In each $E_{j}, j=1, \ldots, 4$, there exists $\rho_{j}>1$ such that for every $1<\tau<\rho_{j}$, there exist functions $\widetilde{v}_{j} \in V_{j}$ such that

$$
\begin{gathered}
\left\|u-\widetilde{v}_{j}\right\|_{0, \Gamma_{j}}=O\left(\tau^{-N_{j}}\right), \\
\left\|\nabla u-\nabla \widetilde{v}_{j}\right\|_{0, \Gamma_{j}}=O\left(\tau^{-N_{j}}\right),
\end{gathered}
$$

for every $1<\tau<\rho_{j}$, as $N_{j} \rightarrow \infty$.

Lemma 5. Let $\varepsilon>0$ and $\widetilde{\varepsilon}>0$ be arbitrarily small. Define $\rho:=R / L$ and $\rho_{\varepsilon}:=\rho-\varepsilon$. Let $\nu$ be the outward normal direction at the disk with radius $R$. Then

$$
\min _{v \in V_{e}}\left(\left\|\partial_{\nu} u_{s}-\partial_{\nu} v\right\|_{0, \Gamma_{R}}^{2}+k^{2}\left\|u_{s}-v\right\|_{0, \Gamma_{R}}^{2}\right)=O\left(\rho_{5}^{-2 N_{5}}\right),
$$

where

$$
\rho_{5}= \begin{cases}\left(\frac{R}{R_{0}}-\widetilde{\varepsilon}\right)^{2}, & \frac{R}{R_{0}}<\rho_{\varepsilon}^{1 / 2} \\ \rho_{\varepsilon}, & \frac{R}{R_{0}}>\rho_{\varepsilon}^{1 / 2} .\end{cases}
$$

Lemma 5 gives the approximation property of the linear combination of half-space Green functions. We omit the proof of this Lemma here, because it is similar to the proof of Theorem 5.4 in [15], which states the approximation property of the linear combination of free-space Green functions.

Assume that there exist $n_{i} \in \mathbb{N}, i=1,2, \ldots, 5 \in \mathbb{N}$, and $N \in \mathbb{N}$ such that $N_{i}, i=1,2, \ldots, 5$, satisfy the following condition:

$$
N_{i}=n_{i} N, \quad i=1,2, \ldots, 5 .
$$

Combining Theorem 1, Proposition 4, and Lemma 5, we immediately obtain the following theorem.

Theorem 6. Let $\rho_{1}, \ldots, \rho_{4}$ be the exponential convergence rates given in Proposition 4 and let $\rho_{5}$ be given in Lemma 5. Defining $\omega:=\min \left\{\rho_{1}^{n_{1}}, \rho_{2}^{n_{2}}, \ldots, \rho_{5}^{n_{5}}\right\}$, then for any $0<\varepsilon<$ $\omega-1$, one has

$$
\left\|u-u_{N}\right\|_{0, \Omega} \leq C(\omega-\varepsilon)^{-N}
$$

\section{Applied to the Inverse Problem}

In this section, we consider the numerical method for solving the inverse scattering problem of rectangular cavity. Uniqueness and local stability results for this problem can be found in [14]. Since the shape of the rectangular cavity can be uniquely determined by three parameters $a, l$, and $h$ (see Figure 1), the inverse problem we considered reduces to reconstructing $a, l$, and $h$ from the near field data on $\Gamma_{R}$. Let $F$ be a nonlinear operator from $\mathbb{R}^{3}$ to $H^{s}\left(\Gamma_{R}\right)(s \geq 1 / 2)$ :

$$
F(a, l, h)=\left.u\right|_{\Gamma_{R}},
$$

where $u$ is the solution to corresponding scattering problem of cavity $D$ which is decided by $a, l$, and $h$. Using operator $F$, the inverse problem can be presented as finding a solution $(a, l, h)$ which satisfies the operator equation (34) by knowing $\left.u\right|_{\Gamma_{R}}$.

We solve operator equation (34) by Newton method. Let $(\partial F / \partial a)(a, l, h),(\partial F / \partial l)(a, l, h)$, and $(\partial F / \partial h)(a, l, h)$ be the Fréchet derivatives of $F$ about $a, l$, and $h$, respectively. According to the results in the literature [17-19], we have $(\partial F / \partial a)(a, l, h)=\left.\psi^{a}\right|_{\Gamma_{R}},(\partial F / \partial l)(a, l, h)=\left.\psi^{l}\right|_{\Gamma_{R}}$, and $(\partial F / \partial h)(a, l, h)=\left.\psi^{h}\right|_{\Gamma_{R}}$, where $\psi^{a}, \psi^{l}$, and $\psi^{h}$ are the solutions to the following differential equations:

$$
\begin{gathered}
\Delta \psi^{a}+k^{2} \psi^{a}=0, \quad \text { in } D \cup \mathbb{R}_{+}^{2}, \\
\psi^{a}=-\frac{\partial u}{\partial x}, \quad \text { on } \Gamma_{a}=\{a, a+l\} \times(-h, 0), \\
\psi^{a}=0, \quad \text { on } \Sigma \backslash \Gamma_{a}, \\
\lim _{r \rightarrow \infty} \sqrt{r}\left(\frac{\partial \psi^{a}}{\partial r}-i k \psi^{a}\right)=0, \quad r=|\mathbf{x}| ;
\end{gathered}
$$




$$
\begin{gathered}
\Delta \psi^{l}+k^{2} \psi^{l}=0, \quad \text { in } D \cup \mathbb{R}_{+}^{2}, \\
\psi^{l}=-\frac{\partial u}{\partial x}, \quad \text { on } \Gamma_{l}:=\{a+l\} \times(-h, 0), \\
\psi^{l}=0, \quad \text { on } \sum \backslash \Gamma_{l}, \\
\lim _{r \rightarrow \infty} \sqrt{r}\left(\frac{\partial \psi^{l}}{\partial r}-i k \psi^{l}\right)=0, \quad r=|\mathbf{x}| ; \\
\Delta \psi^{h}+k^{2} \psi^{h}=0, \quad \text { in } D \cup \mathbb{R}_{+}^{2}, \\
\psi^{h}=-\frac{\partial u}{\partial y}, \quad \text { on } \Gamma_{h}:=(a, a+l) \times\{-h\} \\
\psi^{h}=0, \quad \text { on } \sum \backslash \Gamma_{h}, \\
\lim _{r \rightarrow \infty} \sqrt{r}\left(\frac{\partial \psi^{h}}{\partial r}-i k \psi^{h}\right)=0, \quad r=|\mathbf{x}| .
\end{gathered}
$$

Remark 7. The structures of (35), (36), and (37) are similar to the structure of (2), so we can use least-squares finite element method to solve (35), (36), and (37) which can be viewed as some particular direct scattering problems.

Remark 8. For the deduction of (35), (36), and (37), we refer to $[13,17-19]$. The idea is that first we give a perturbation of the original geometry of the cavity (small variation of $a$, $l$, or $h$ ) and get a new scattering problem of the new cavity. Then we build the map between the original geometry and the new geometry. Using this map we transform the new cavity domain to the original one and then we can get the variational formulation of the two problems in the same domain. At last, we obtain the difference between the original and new scattered fields and then get the domain derivatives by taking the variations of the geometry parameters tending to 0 .

Since the least-squares nonpolynomial finite element method is very efficient, we solve $(\partial F / \partial a)(a, l, h)$, $(\partial F / \partial l)(a, l, h)$, and $(\partial F / \partial h)(a, l, h)$ by this method. Because the above boundary condition contains the solution $u$ to the direct problem, we assume $u_{N}$ is the numerical solution of $u$ by least-squares finite element method, and then we substitute $u$ with $u_{N}$ in the boundary condition. When solving (35), we use the same domain decompositions as solving $u$. In subdomain $E_{5}$, we choose the approximation space $\widetilde{V}_{5}$ as follows:

$$
\begin{aligned}
\widetilde{V}_{5}=\{v & \in L_{\text {loc }}^{2}\left(E_{5}\right), \\
v & \left.=\sum_{j=1}^{N_{5}-1} c_{j}^{(5)}\left(\frac{\partial}{\partial n\left(\mathbf{y}_{j}\right)} G\left(\mathbf{x}, \mathbf{y}_{j}\right)+i k G\left(\mathbf{x}, \mathbf{y}_{j}\right)\right)\right\} ;
\end{aligned}
$$

In each subdomain of $E_{1}, E_{2}, E_{3}$, and $E_{4}$, we choose space

$$
\begin{array}{r}
V_{j}^{a} \\
=\left\{v \in L^{2}\left(E_{j}\right), v=-\frac{\partial u_{N}}{\partial x}+\sum_{n=1}^{N_{j}} c_{n}^{(j)} J_{\gamma_{j} n}(k r) \sin \left(\gamma_{j} n \theta\right)\right\}, \\
j=1,2,3,4 .
\end{array}
$$

The functions in $V_{j}^{a}$ satisfy Helmholtz equation and boundary condition on $\Sigma \backslash \Gamma^{a}$ strictly. They also approximately satisfy the boundary condition on $\Gamma^{a}$.

Define nonpolynomial finite element space $V^{a}$ as follows:

$$
\begin{gathered}
V^{a}=\left\{v \in L_{\text {loc }}^{2}\left(R_{+}^{2} \cup D\right),\left.v\right|_{E_{j}} \in V_{j}^{a},\right. \\
\left.j=1,2,3,4,\left.v\right|_{E_{5}} \in \widetilde{V}_{5}\right\} .
\end{gathered}
$$

Then the approximation of $\psi_{N}^{a}$ to $\psi^{a}$ by least-squares finite element method can be defined as the solution to the following least-squares problem:

$$
\psi_{N}^{a}=\arg \min _{v \in V^{a}} J(v) .
$$

We recall (9) for the definition of $J(v)$. The technique used for solving (36) and (37) is similar to the above. So we omit the details here.

From the above analysis, the steps of Newton method for solving (34) are as follows.

Step 1. Let $n=0$ and $\left(a_{n}, l_{n}, h_{n}\right)$ be an initial value of $(a, l, h)$.

Step 2. Use least-squares nonpolynomial finite element method to solve the scattering problem defined by $\left(a_{n}, l_{n}, h_{n}\right)$ to get the numerical solutions $u_{n, N}$ and $\left.u_{n, N}\right|_{\Gamma_{R}}$.

Step 3. Compute $(\partial F / \partial a)\left(a_{n}, l_{n}, h_{n}\right),(\partial F / \partial l)\left(a_{n}, l_{n}, h_{n}\right)$, and $(\partial F / \partial h)\left(a_{n}, l_{n}, h_{n}\right)$ by least-squares nonpolynomial finite element method.

Step 4. Use $u_{n, N}$ from Step 2 to solve $\left(\delta_{a_{n}}, \delta_{l_{n}}, \delta_{h_{n}}\right)$ to minimize

$$
\begin{gathered}
\|\left. u\right|_{\Gamma_{R}}-\left(\left.u_{n}\right|_{\Gamma_{R}}+\frac{\partial F}{\partial a}\left(a_{n}, l_{n}, h_{n}\right) \delta_{a_{n}}+\frac{\partial F}{\partial l}\left(a_{n}, l_{n}, h_{n}\right) \delta_{l_{n}}\right. \\
\left.+\frac{\partial F}{\partial h}\left(a_{n}, l_{n}, h_{n}\right) \delta_{h_{n}}\right) \|_{0, \Gamma_{R}} .
\end{gathered}
$$

Step 5. Set $\left(a_{n+1}, l_{n+1}, h_{n+1}\right)=\left(a_{n}, l_{n}, h_{n}\right)+\left(\delta_{a_{n}}, \delta_{l_{n}}, \delta_{h_{n}}\right)$.

Step 6. Let $n=n+1$, and repeat Step 2 to Step 5 until some stopping rule is satisfied.

\section{Numerical Examples}

In this section we demonstrate the numerical results of our method on the direct and inverse scattering problems for 
rectangular cavity. All computations were performed using MATLAB.

Example 1. Assume the open cavity $D=(-1,1) \times(-2,0)$. The artificial boundary $\Gamma_{R}=\left\{\mathbf{x} \in \mathbb{R}_{+}^{2} ;|\mathbf{x}|=2\right\}$, and the source points lie on $\Gamma_{R_{0}}=\left\{\mathbf{x} \in \mathbb{R}_{+}^{2} ;|\mathbf{x}|=1.6\right\}$. The incident wave is given by $u_{i}=e^{i k x \sin \theta-i k y \cos \theta}$ with $k=5$ and $\theta=-\pi / 4$.

We choose $N_{i}=N, i=1,2, \ldots, 5$. In the left plot of Figure 3, we show the convergence results for the matching error functional $J\left(u_{N}\right)$. We plot the curve of $\log J\left(u_{N}\right)^{1 / 2}$ versus $N$ and the estimate convergence rate $\log \omega^{-N}$, with $\omega \approx 1.3457$. In the right plot of Figure 3, we show the real part of the approximate total field $u_{N}$ with $N=50$, and the corresponding $J\left(u_{N}\right)^{1 / 2}=6.4159 \times 10^{-12}$. We can see that the convergence rate of our algorithm is fast and the precision is high. Next we set wave number $k=100$ to show that our method can deal well with scattering problem with large wave number. The angle of incidence is set to be $\theta=-\pi / 4$. Numerical result is shown in Figure 4 with $N=300$, and the corresponding $J\left(u_{N}\right)^{1 / 2}=2.7438 \times 10^{-4}$. The convergence results of the $L^{2}$ error are not shown here, since it is difficult to get the analytical expression of the total field $u$.

In Sections 2 and 3, the artificial boundary $\Gamma_{R}$ is chosen to be a semicircle; see Example 1. Next, in Example 2, we choose the artificial boundary to be a semiellipse. If the cavity is wide, using a semicircle as artificial boundary will lead to a large computational domain, while a properly chosen ellipse artificial boundary will lead to a small computational domain. Though the theoretical analysis of using a semiellipse as artificial boundary is not given in this paper, we will give a numerical example to show that elliptic artificial boundary works well. The theoretical analysis for elliptic artificial boundary will be our future consideration.

Example 2. Assume the wide open cavity $D=(-2,2) \times$ $(-2,0)$. The artificial boundary $\Gamma_{e}=\left\{(x, y) \in \mathbb{R}_{+}^{2} ; x=\right.$ $3 \cos t, y=\sin t, t \in[0, \pi]\}$, and the source points lie on $\Gamma_{f}=\left\{(x, y) \in \mathbb{R}_{+}^{2} ; x=2.5 \cos t, y=0.5 \sin t, t \in[0, \pi]\right\}$. The wave number is $k=10$, and the angle of incidence is $\theta=-\pi / 4$.

In the left plot of Figure 5, we show the domain decomposition for the wide cavity. In the right plot of Figure 3, we show the real part of the approximate total field $u_{N}$ with $N=50$ and the corresponding $J\left(u_{N}\right)^{1 / 2}=1.0552 \times 10^{-6}$.

Example 3. In this example, we will show the numerical results of our method for the inverse problem. Assume the open cavity $D=(-1,1) \times(-2,0)$; that is, $a=-1, l=2$, and $h=2$. The total field $u$ is measured on the curve $\Gamma_{R}=\{\mathbf{x} \in$ $\left.\mathbb{R}_{+}^{2} ;|\mathbf{x}|=2\right\}$. Then we use the measured field to reconstruct the three parameters $a, l$, and $h$. We choose wave numbers $k=1.5$ and $k=10$ and angle of incidence $\theta=0$. The reconstructed results are shown in Tables $1 \sim 4$, where $n$ is the iterative step. Tables 1 and 3 show the results of noise-free
TABLE 1: Reconstructed result without noise: $k=1.5 ; \theta=0$.

\begin{tabular}{cccc}
\hline$n$ & $a_{n}$ & $l_{n}$ & $h_{n}$ \\
\hline 0 & -1.2 & 1.4 & 2.2 \\
1 & -1.007658741131911 & 1.992109594216756 & 1.686727120231955 \\
2 & -0.995217523024954 & 1.991608675610521 & 2.035120388009096 \\
3 & -0.999972332726015 & 1.999935535112317 & 1.999225457890670
\end{tabular}

TABLE 2: Reconstructed result with 5\% relative noise: $k=1.5 ; \theta=0$.

\begin{tabular}{cccc}
\hline$n$ & $a_{n}$ & $l_{n}$ & $h_{n}$ \\
\hline 0 & -1.2 & 1.4 & 2.2 \\
1 & -1.007341847307904 & 1.988937204297816 & 1.698647382173970 \\
2 & -0.994072787628460 & 1.986459820750302 & 2.095129045530898 \\
3 & -0.999204833909075 & 2.070910501205903 & 1.995541525403421
\end{tabular}

cases. Then we add 5\% random relative noise to the scattering fields, and the corresponding results are shown in Tables 2 and 4 . Here, the relative noise is defined as $\left\|u-u^{\delta}\right\|_{2} /\|u\|_{2}$, where $u^{\delta}$ is the random perturbation of $u$. We can see that our algorithm works well in all cases.

Example 4. Assume the open cavity $D=(-2,2) \times(-2,0)$; that is, $a=-2, l=4$, and $h=2$. The total field $u$ is measured on the curve $\Gamma_{R}=\left\{\mathbf{x} \in \mathbb{R}_{+}^{2} ;|\mathbf{x}|=5\right\}$. We choose wave numbers $k=10$ and $k=50$. The reconstructed results are shown in Tables $5 \sim 8$. For space constraints, we only present partial iterated steps in Tables 7 and 8.

From the results of Examples 3 and 4, we can see that with the increment of wave number the reconstructed results become more sensitive to initial values. This is due to the local convergence of Newton method.

\section{Conclusions}

In this paper we use the least-squares nonpolynomial finite element method to solve the direct scattering problem for rectangular open cavity and give an $L^{2}$ error estimate for the method. Due to the exponential convergence, our method is efficient and highly accurate. So it is very competitive for intermediate or high frequency problems. This is also presented by our numerical examples. Furthermore, the use of fractional Fourier-Bessel basis functions deals well with the singularities of the solution. We also give an algorithm for solving the inverse scattering problem for rectangular cavity by coupling the least-squares nonpolynomial finite element method and the Newton method. And numerical examples verify that our method works well with the inverse problem.

\section{Conflict of Interests}

The authors declare that there is no conflict of interests regarding the publication of this paper. 


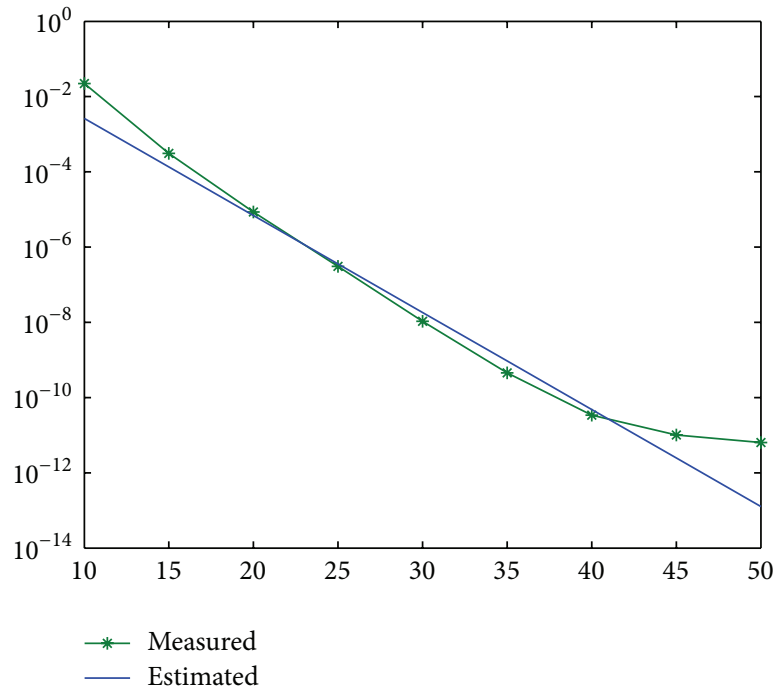

(a)

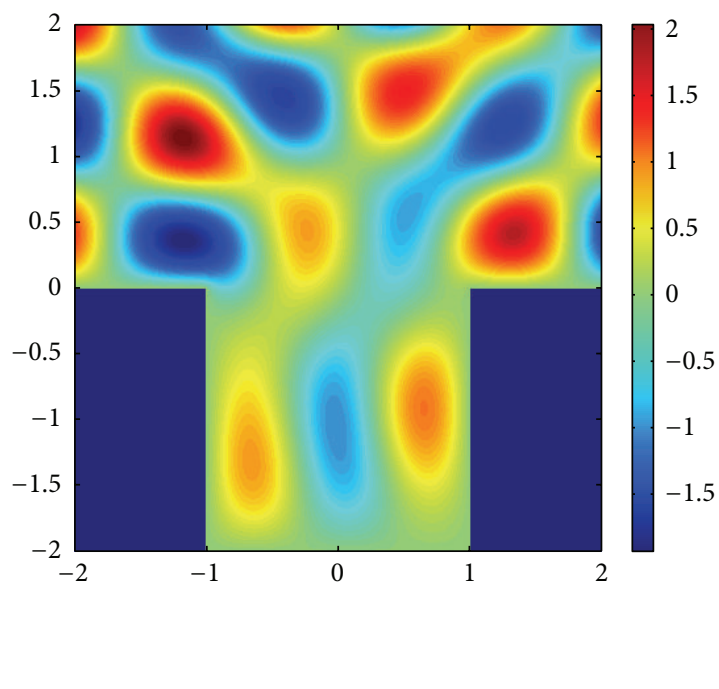

(b)

FIGURE 3: Rectangular cavity scattering. (a) The convergence of $J\left(u_{N}\right)$ with respect to $N$. (b) The real part of the total field $u$.

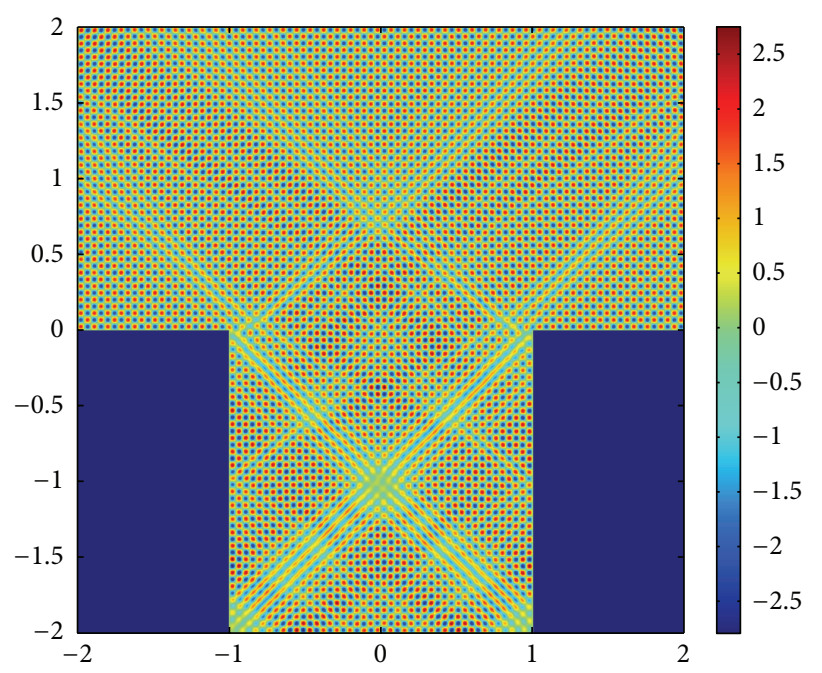

FIGURE 4: Real part of the total field $u$ with $k=100$ and $\theta=-\pi / 4$.

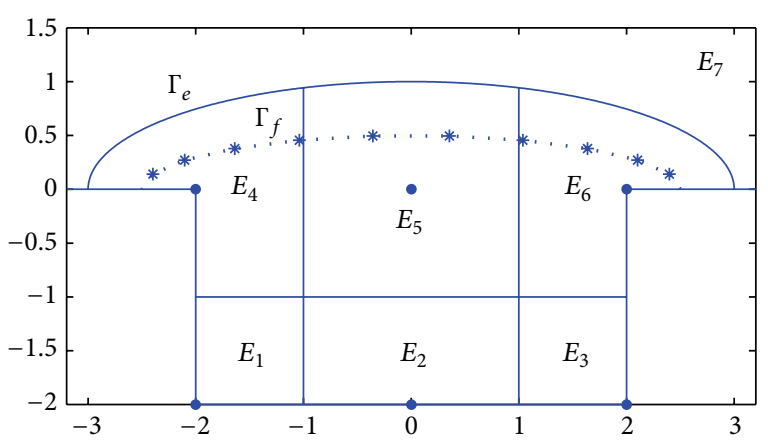

(a)

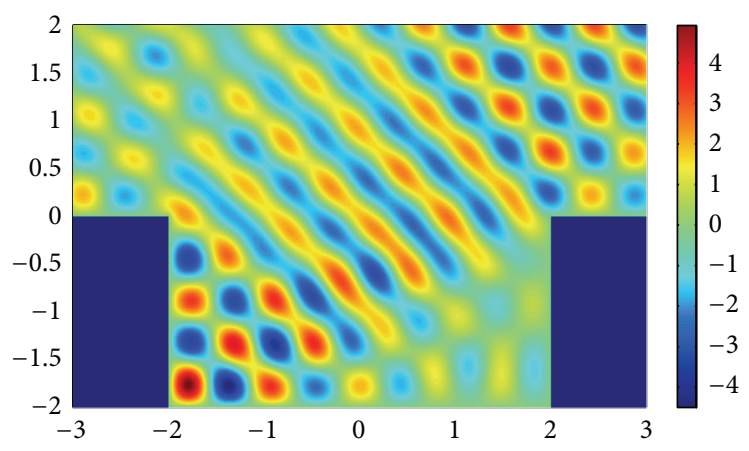

(b)

FIGURE 5: Wide rectangular cavity scattering. (a) Decomposition of the cavity. (b) The real part of the total field $u$. 
TABLE 3: Reconstructed result without noise: $k=10 ; \theta=0$.

\begin{tabular}{lccc}
\hline$n$ & $a_{n}$ & $l_{n}$ & $h_{n}$ \\
\hline 0 & -1.2 & 2.1 & 2.2 \\
1 & -1.182799697846479 & 2.063369519964419 & 2.177437266265451 \\
2 & -1.149484316496610 & 2.020324785735872 & 2.153775419824001 \\
3 & -1.093795718419669 & 1.999503403288712 & 2.107212983007467 \\
4 & -1.032253885993330 & 1.998568377066760 & 2.040534190205991 \\
5 & -1.001747960610638 & 1.999820195805721 & 2.003005533213011 \\
6 & -0.999997247632396 & 1.999999589257117 & 1.999996865650199 \\
7 & -0.999999999991650 & 1.999999999999873 & 1.999999999995763 \\
\hline
\end{tabular}

TABLE 4: Reconstructed result with 5\% relative noise: $k=10 ; \theta=0$.

\begin{tabular}{lccc}
\hline$n$ & $a_{n}$ & $l_{n}$ & $h_{n}$ \\
\hline 0 & -1.2 & 2.1 & 2.2 \\
1 & -1.182582978678448 & 2.063551103051895 & 2.177320775165475 \\
2 & -1.148350997143566 & 2.020607260524955 & 2.153119170884156 \\
3 & -1.091941880923172 & 1.999548335908778 & 2.106277154627640 \\
4 & -1.030938972103234 & 1.998488304897653 & 2.040506699619247 \\
5 & -1.001742260952702 & 1.999681386603467 & 2.004495721419121 \\
6 & -0.999621718913080 & 1.999872216653859 & 2.001068706129402 \\
7 & -0.999564224922524 & 1.999876119900957 & 2.000996585243444 \\
8 & -0.999562879905584 & 1.999876193707215 & 2.000994921602748 \\
\hline
\end{tabular}

TABLE 5: Reconstructed result without noise: $k=10 ; \theta=-\pi / 6$.

\begin{tabular}{lccc}
\hline$n$ & $a_{n}$ & $l_{n}$ & $h_{n}$ \\
\hline 0 & -1.9 & 1.9 & 4.1 \\
1 & -1.895201991129818 & 1.936076943236395 & 4.067911959164771 \\
2 & -1.931940394007886 & 1.972231481981323 & 4.018004942923492 \\
3 & -1.995306222963697 & 1.992128483183547 & 4.012291393206398 \\
4 & -2.000306191593380 & 1.999830393801205 & 4.000309420921332 \\
5 & -1.999999881701120 & 2.000000013296713 & 3.999999774941242 \\
6 & -2.000000000034648 & 1.999999999984548 & 4.000000000009253 \\
\hline
\end{tabular}

TABLE 6: Reconstructed result with $5 \%$ relative noise: $k=10 ; \theta=-\pi / 6$.

\begin{tabular}{lccc}
\hline$n$ & $a_{n}$ & $l_{n}$ & $h_{n}$ \\
\hline 0 & -1.9 & 1.9 & 4.1 \\
1 & -1.895838941855006 & 1.935894425740055 & 4.067042714936927 \\
2 & -1.932097432298987 & 1.972152124270138 & 4.016200758052248 \\
3 & -1.994328852044417 & 1.992079891039202 & 4.010768960405867 \\
4 & -1.999797197477044 & 1.999676078163155 & 3.999427449734399 \\
5 & -1.999487297516183 & 1.999916869790411 & 3.999100323168048 \\
6 & -1.999483071762259 & 1.999919457393489 & 3.999095572355909 \\
\hline
\end{tabular}

TABLE 7: Reconstructed result without noise: $k=50 ; \theta=-\pi / 6$.

\begin{tabular}{lccc}
\hline$n$ & $a_{n}$ & $l_{n}$ & $h_{n}$ \\
\hline 0 & -2.03 & 1.97 & 4.03 \\
2 & -2.031535181558714 & 1.981928779622648 & 4.029171981244078 \\
4 & -2.005568849168570 & 1.997581097942630 & 4.021193787092940 \\
6 & -2.001974139433433 & 1.999148310000414 & 4.012618600367861 \\
8 & -2.000511548627857 & 1.999827799707791 & 4.002594529162001 \\
10 & -2.000003015153972 & 1.999998442763691 & 4.000009099104641 \\
12 & -2.000000000489355 & 2.000000001041293 & 4.000000009657497 \\
\hline
\end{tabular}


TABLE 8: Reconstructed result with $5 \%$ relative noise: $k=50 ; \theta=-\pi / 6$.

\begin{tabular}{lccc}
\hline$n$ & $a_{n}$ & $l_{n}$ & $h_{n}$ \\
\hline 0 & -2.03 & 1.97 & 4.03 \\
2 & -2.031842498312580 & 1.981759352432498 & 4.029112488060880 \\
4 & -2.005863473406319 & 1.997424185260155 & 4.020032914816695 \\
6 & -2.001608041761260 & 1.999301145363739 & 4.009438148872054 \\
8 & -2.000228300961412 & 1.999901659431604 & 4.001097042616364 \\
10 & -2.000054941166881 & 1.999955848956634 & 4.000575196317175 \\
12 & -2.000055138883032 & 1.999955773509758 & 4.000575517974161 \\
\hline
\end{tabular}

\section{Acknowledgments}

The first author was supported by the Fundamental Research Funds for the Central Universities 3132014038. The second and third authors were supported by NSFC Grant 11371172. The authors would like to thank the anonymous referees for many constructive comments and suggestions.

\section{References}

[1] H. Ammari, G. Bao, and A. W. Wood, "Analysis of the electromagnetic scattering from a cavity," Japan Journal of Industrial and Applied Mathematics, vol. 19, no. 2, pp. 301-310, 2002.

[2] H. Ammari, G. Bao, and A. W. Wood, "A cavity problem for Maxwell's equations," Methods and Applications of Analysis, vol. 9, no. 2, pp. 249-259, 2002.

[3] H. Ammari, G. Bao, and A. W. Wood, "An integral equation method for the electromagnetic scattering from cavities," Mathematical Methods in the Applied Sciences, vol. 23, no. 12, pp. 1057-1072, 2000.

[4] T.-T. Chia, R. J. Burkholder, and R. Lee, "Application of FDTD in hybrid methods for cavity scattering analysis," IEEE Transactions on Antennas and Propagation, vol. 43, no. 10, pp. 10821090, 1995.

[5] J.-M. Jin and J. L. Volakis, "A hybrid finite element method for scattering and radiation by microstrip patch antennas and arrays residing in a cavity," IEEE Transactions on Antennas and Propagation, vol. 39, no. 11, pp. 1598-1604, 1991.

[6] D. C. Ross, J. L. Volakis, and H. T. Anastassiu, "Threedimensional edge-based finite-element analysis for discrete bodies of revolution," IEEE Transactions on Antennas and Propagation, vol. 45, no. 7, pp. 1160-1165, 1997.

[7] A. Barka, P. Soudais, and D. Volpert, "Scattering from 3-D cavities with a plug and play numerical scheme combining IE, PDE, and modal techniques," IEEE Transactions on Antennas and Propagation, vol. 48, no. 5, pp. 704-712, 2000.

[8] J.-M. Jin, S. S. Ni, and S.-W. Lee, "Hybridization of SBR and FEM for scattering by large bodies with cracks and cavities," IEEE Transactions on Antennas and Propagation, vol. 43, no. 10, pp. 1130-1139, 1995.

[9] D. C. Ross, J. L. Volakis, and H. T. Anastassiu, "Hybrid finite element-modal analysis of jet engine inlet scattering," IEEE Transactions on Antennas and Propagation, vol. 43, no. 3, pp. 277-285, 1995.

[10] G. Bao, K. Yun, and Z. Zhou, "Stability of the scattering from a large electromagnetic cavity in two dimensions," SIAM Journal on Mathematical Analysis, vol. 44, no. 1, pp. 383-404, 2012.
[11] H. Li, H. Ma, and W. Sun, "Legendre spectral Galerkin method for electromagnetic scattering from large cavities," SIAM Journal on Numerical Analysis, vol. 51, no. 1, pp. 353-376, 2013.

[12] L. Feng and F. Ma, "Uniqueness and local stability for the inverse scattering problem of determining the cavity," Science in China Series A: Mathematics, vol. 48, no. 8, pp. 1113-1123, 2005.

[13] J. Liu, "On uniqueness and linearization of an inverse electromagnetic scattering problem," Applied Mathematics and Computation, vol. 171, no. 1, pp. 406-419, 2005.

[14] G. Bao, J. Gao, and P. Li, "Analysis of direct and inverse cavity scattering problems," Numerical Mathematics. Theory, Methods and Applications, vol. 4, no. 3, pp. 335-358, 2011.

[15] A. H. Barnett and T. Betcke, "An exponentially convergent nonpolynomial finite element method for time-harmonic scattering from polygons," SIAM Journal on Scientific Computing, vol. 32, no. 3, pp. 1417-1441, 2010.

[16] P. Monk and D.-Q. Wang, "A least-squares method for the Helmholtz equation," Computer Methods in Applied Mechanics and Engineering, vol. 175, no. 1-2, pp. 121-136, 1999.

[17] J. Simon, "Differentiation with respect to the domain in boundary value problems," Numerical Functional Analysis and Optimization, vol. 2, no. 7-8, pp. 649-687 (1981), 1980.

[18] R. Djellouli, C. Farhat, J. Mandel, and P. Vanek, "A stability estimate for direct acoustic scattering problems by differentiation with respect to the obstacle domain," IMA Journal of Applied Mathematics, vol. 2, pp. 1-19, 1999.

[19] R. Djellouli and C. Farhat, "On the characterization of the Fréchet derivative with respect to a Lipschitz domain of the acoustic scattered field," Journal of Mathematical Analysis and Applications, vol. 238, no. 1, pp. 259-276, 1999.

[20] M. Stojek, "Least-squares Trefftz-type elements for the Helmholtz equation," International Journal for Numerical Methods in Engineering, vol. 41, no. 5, pp. 831-849, 1998.

[21] T. Betcke, "A GSVD formulation of a domain decomposition method for planar eigenvalue problems," IMA Journal of Numerical Analysis, vol. 27, no. 3, pp. 451-478, 2007. 


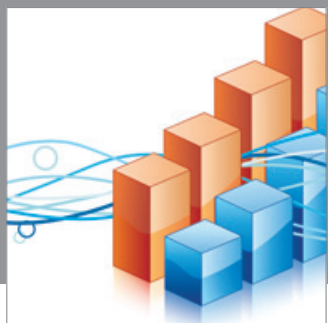

Advances in

Operations Research

mansans

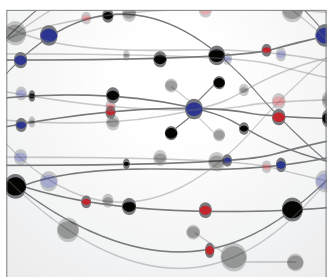

The Scientific World Journal
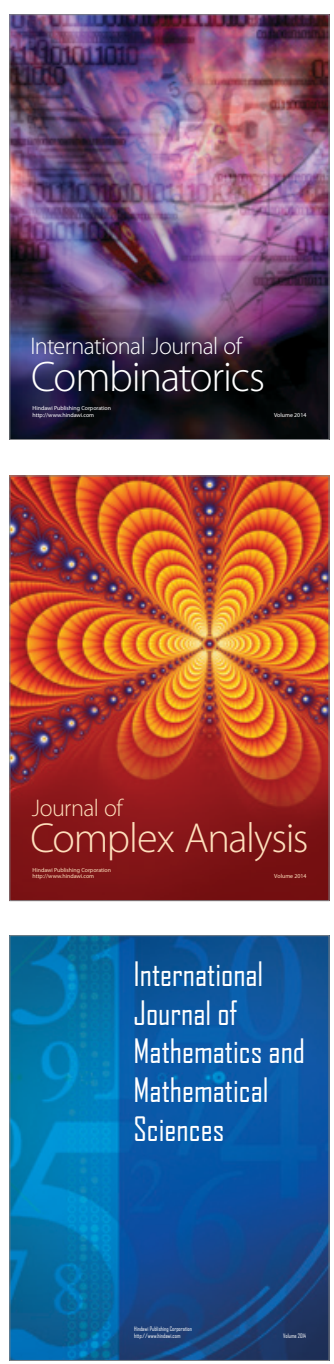
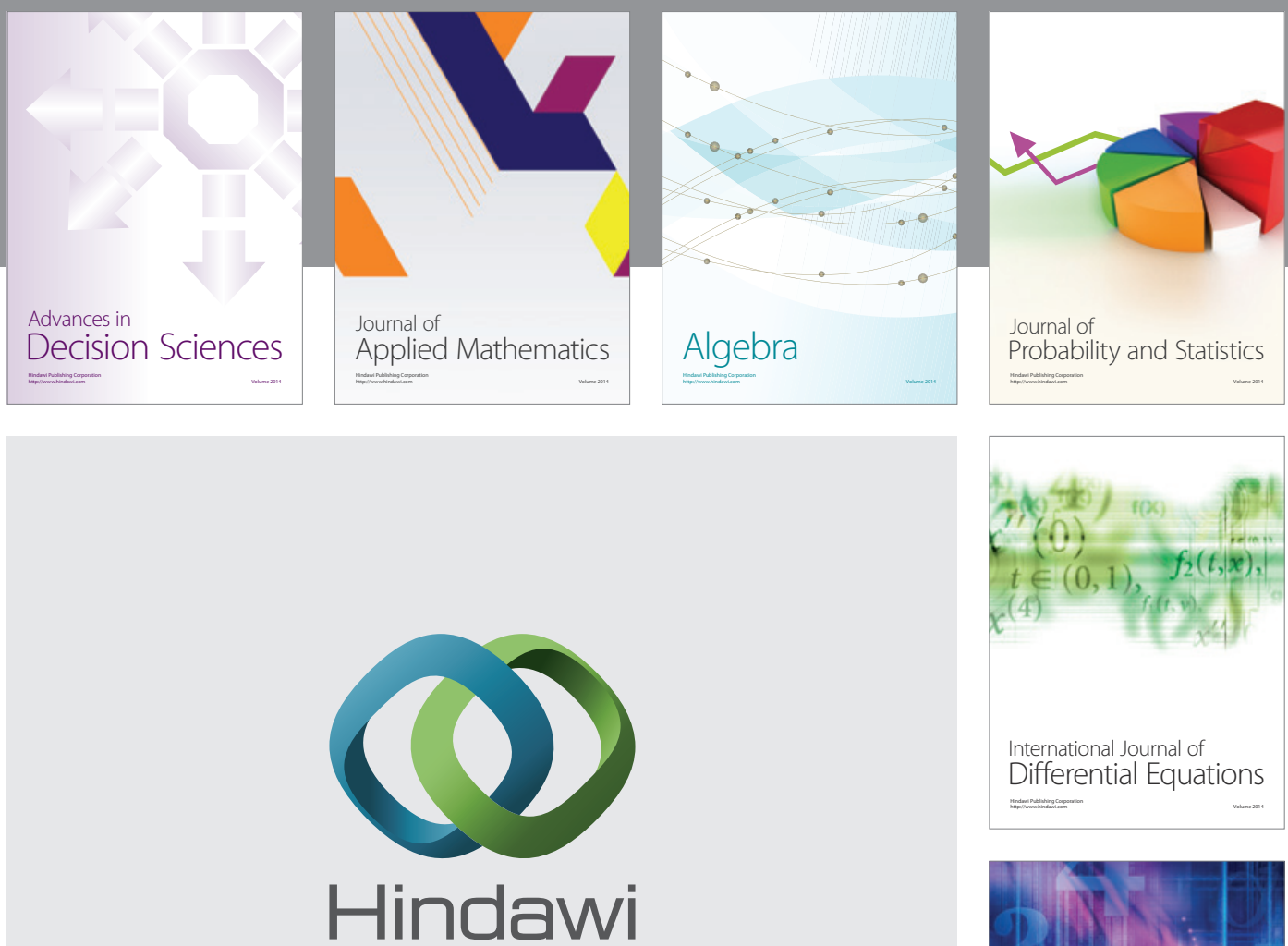

Submit your manuscripts at http://www.hindawi.com
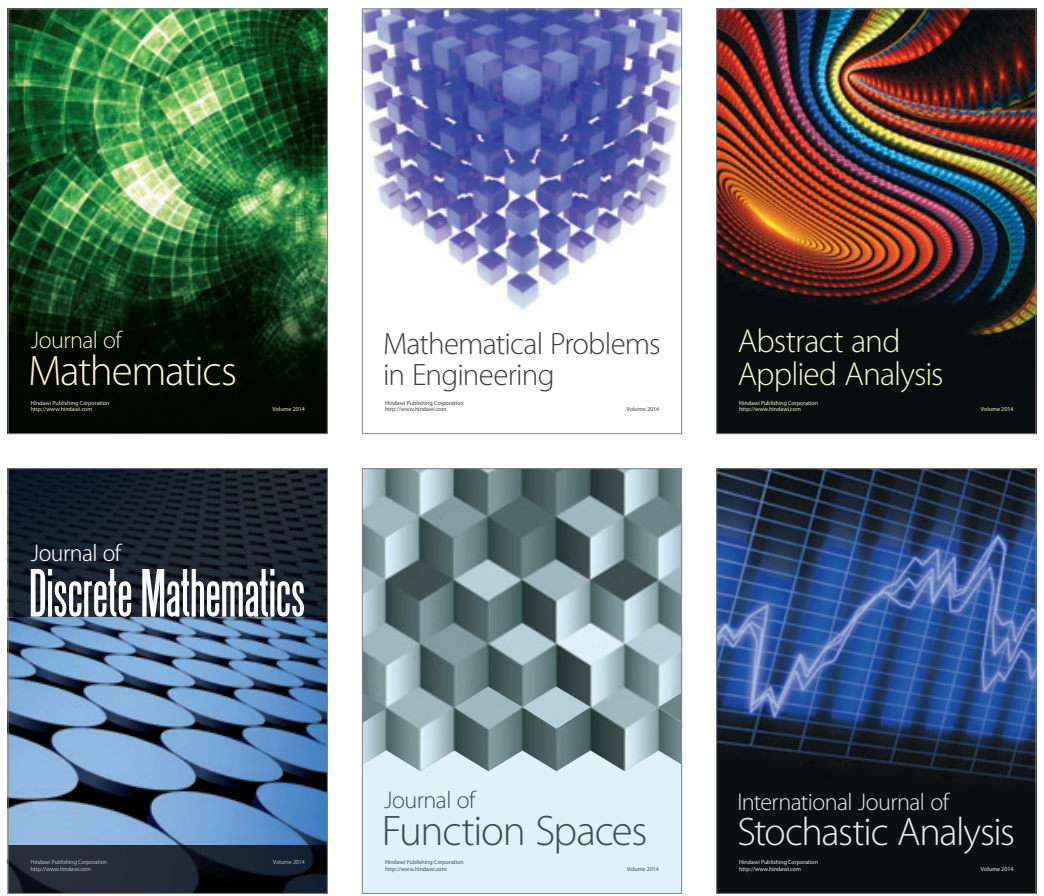

Journal of

Function Spaces

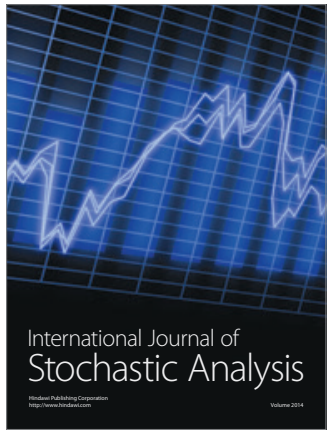

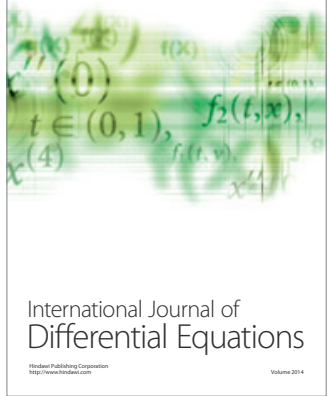
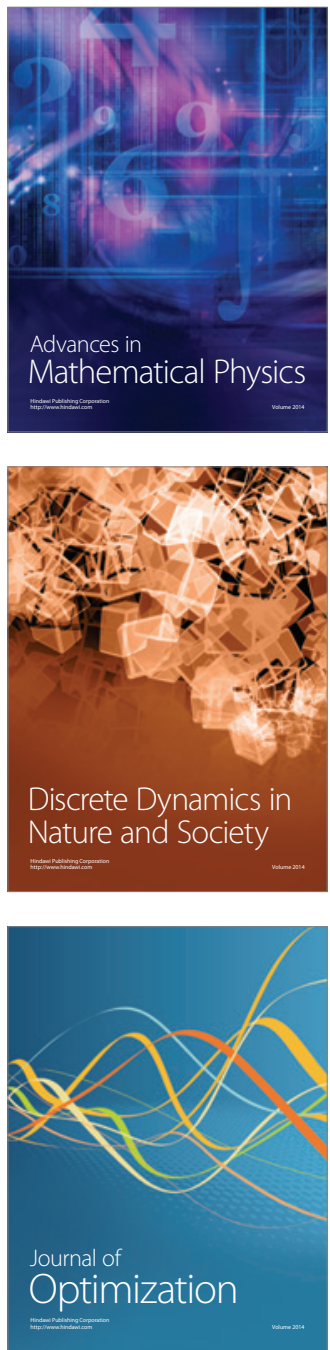